\section{El VIH/sida en el lugar de trabajo. Recomendaciones prácticas de la OIT $^{1}$}

Palabras clave: VIH/sida, salud ocupacional, OIT.

\footnotetext{
1 Basado en el documento Repertorio de recomendaciones prácticas de la OIT sobre el VIH/sida y el mundo del trabajo, elaborado por la Organización Internacional del Trabajo (Ginebra, 2001). Disponible en versión electrónica, a texto completo en español, en http://www.ilo. org/public/english/protection/trav/aids/pdf/acodesp.pdf
}

En la actualidad hay unos 36 millones de personas infectadas por el VIH/sida. Se estima que, de ellas, más de 23 millones son personas económicamente activas entre los 15 y 49 años de edad. Por lo menos 17,5 millones se encuentran en 43 países africanos, donde, según declaraciones de la Organización Internacional del Trabajo (OIT), la epidemia del VIH ha creado "un estado de emergencia". En América Latina y el Caribe esta cifra asciende a 642000 casos.

Todas estas personas representan una fuerza de trabajo mermada por los efectos devastadores de la enfermedad, dado que, al flagelo humano y social que entraña, el VIH/sida agrega una carga económica extraordinaria, pues ataca a los miembros de la sociedad más productivos. En lo que se refiere al mundo del trabajo, el VIH/sida reduce los ingresos, impone costos mayores a las empresas y menoscaba derechos laborales fundamentales debido a la discriminación y al rechazo que sufren las personas infectadas. Más aún, la epidemia aqueja con mayor crueldad a los colectivos vulnerables, lo que deriva en graves problemas adicionales, como el aumento de las desigualdades entre hombres y mujeres y la exacerbación del trabajo infantil.

Estas son las razones que llevaron a la OIT a manifestarse enérgicamente por medio de un documento titulado Repertorio de recomendaciones prácticas sobre el VIH/sida y el mundo del trabajo. En palabras del Director General de la OIT, Juan Somavia, "el Repertorio no se reduce simplemente a políticas y directrices, sino que se refiere también al respeto de la dignidad de los demás y a la necesidad de aprender a vivir con la realidad del VIH/sida [...] a nuestro lado".

\section{PROPÓSITO}

El Repertorio tiene el propósito de ayudar a hacer frente a la epidemia del VIH/sida en el mundo del trabajo, mediante un conjunto de directrices relacionadas con: a) la prevención del VIH/ sida; b) la gestión y atenuación de sus efectos en el mundo del trabajo; c) la prestación de asistencia y apoyo a los trabajadores infectados y, en general, a todas las personas afectadas por esta epidemia; y d) la erradicación de la discriminación contra la persona real o supuestamente infectada por el virus. 


\section{UTILIDAD Y ALCANCE}

El Repertorio ha sido creado como un instrumento destinado a contribuir a elaborar y adoptar medidas específicas en el lugar de trabajo, promoviendo el diálogo y otras formas de cooperación entre el gobierno, los empleadores, los trabajadores y sus representantes, el personal de salud en el trabajo, los especialistas en VIH/sida y otras partes interesadas, tales como organizaciones no gubernamentales y organizaciones de la sociedad civil. La idea es que sirva también para poner en práctica sus recomendaciones, integrándolas en leyes, políticas y programas nacionales; convenios de empresa o de establecimiento, y políticas y planes de acción en el lugar de trabajo. Su contenido se aplica a todos los empleadores y trabajadores (incluidas las personas que buscan empleo) de los sectores público y privado y a todas las formas de trabajo, sea formal o informal.

\section{PRINCIPIOS FUNDAMENTALES}

\section{Reconocimiento del problema}

El VIH/sida constituye un problema en el lugar de trabajo y debería tratarse como cualquier otra enfermedad profesional grave, no solo porque afecta a los trabajadores sino también porque, al ser el lugar de trabajo parte integrante de la vida local, le corresponde una misión importante en la lucha general contra la propagación y los efectos de la epidemia.

\section{La discriminación}

Con arreglo al principio de trabajo decente y respeto de los derechos humanos, no debería haber discriminación alguna contra los trabajadores basada en una infección por el VIH real o sospechada. La discriminación y el rechazo hacia las personas que padecen $\mathrm{VIH} /$ sida entorpecen peligrosamente los esfuerzos por prevenir la epidemia.

\section{Igualdad entre hombres y mujeres}

Es preciso reconocer los problemas de género con respecto al VIH/sida. Las mujeres están más expuestas a la infección y con frecuencia resultan más gravemente afectadas que los hombres debido a razones biológicas, socioculturales y económicas. Cuanto mayor sea la discriminación de género y más baja la posición de las mujeres en la escala social, más perniciosos serán para ellas los efectos del VIH. Por lo tanto, es necesario lograr relaciones de género más igualitarias que ayuden a prevenir eficazmente la propagación de esta enfermedad entre las mujeres.

\section{Un ambiente sano}

La transmisión del VIH solo puede prevenirse en un ambiente de trabajo sano y seguro para todas las partes. De esta manera se promueve una salud física y mental óptima, así como la adaptación del medio laboral a las facultades de los trabajadores en función de su estado de salud.

\section{El diálogo social}

La aplicación adecuada de una política contra el VIH/sida exige colaboración entre los empleadores, los trabajadores y sus representantes. También son importantes la intervención del gobierno y la participación activa de los trabajadores afectados por la epidemia.

\section{Confidencialidad}

No se debería pedir a los solicitantes de un puesto de trabajo, ni a quienes ya se desempeñan en un empleo, que proporcionen información personal relativa al VIH. Tampoco hay razón para obligar a los trabajadores a dar a conocer información de esta índole referente a un compañero. El acceso a los datos personales sobre la posible seropositividad de un trabajador debería regirse por normas de estricta confidencialidad.

\section{Permanencia en el empleo}

La infección por VIH no constituye una causa justificada de despido. Tal como sucede con otras enfermedades, las personas con trastornos derivados del VIH deberían tener la posibilidad de trabajar en un puesto apropiado ya existente mientras sean médicamente aptas para hacerlo.

\section{Prevención}

La infección por VIH puede prevenirse con diversas estrategias que se amolden a las condiciones locales y respeten las características culturales. Dichas estrategias abarcan cambios del comportamiento, la difusión de conocimientos sobre la enfermedad, el tratamiento de personas infectadas y la instauración de un ambiente sin discriminación. 


\section{DERECHOS Y DEBERES}

\section{Los gobiernos y las autoridades competentes}

Compromiso. Los gobiernos deberían incorporar el mundo del trabajo en la estrategia y los programas nacionales contra el VIH/sida, garantizando la participación de representantes de los empleadores, los trabajadores, las personas con VIH/sida y los ministerios responsables de los asuntos laborales y sociales.

Participación multisectorial. Las autoridades competentes deberían impulsar acuerdos de colaboración para prevenir el VIH/sida, en los que participen organismos públicos, el sector privado, las organizaciones de empleadores y de trabajadores y otras partes interesadas.

Directrices médicas. En los países donde los empleadores asumen la responsabilidad de proporcionar directamente atención de salud a los trabajadores, los poderes públicos deberían prestarles apoyo elaborando directrices sanitarias y clínicas sobre el $\mathrm{VIH} /$ sida, en consonancia con los servicios existentes.

Protección social. Los gobiernos deberían procurar que las prestaciones estipuladas en la legislación nacional para los trabajadores afectados por el VIH/ sida no sean inferiores a los de los trabajadores víctimas de otras enfermedades graves. Al concebir y llevar a cabo los programas de seguridad social, los poderes públicos deberían adaptarlos al carácter progresivo e intermitente de la enfermedad, por ejemplo, facilitando las prestaciones cuando y como sean necesarias y asegurando tramitaciones rápidas.

Investigación. Con objeto de lograr coherencia con los programas nacionales contra el sida, calcular el costo de la epidemia en los lugares de trabajo y facilitar una planificación destinada a mitigar su impacto socioeconómico, las autoridades competentes deberían promover y llevar a cabo investigaciones sobre proyecciones demográficas, estudios de incidencia y prevalencia, así como estudios de caso de buenas prácticas. En lo posible, el acopio de datos tendría que hacerse por sectores y desglosarse en función del sexo, raza, preferencia sexual, edad y situación en el empleo u ocupación.

Recursos financieros. De ser posible, los gobiernos, en consulta con los interlocutores sociales y otras partes interesadas, deberían hacer una estimación de las consecuencias financieras del VIH/ sida y esforzarse por conseguir fondos de fuentes locales e internacionales para sus planes nacionales contra el sida y para su sistema de seguridad social. Asimismo, cuando los gobiernos aportan fondos $u$ otro tipo de subvenciones para el establecimiento de empresas, deberían exigirles que acaten lo dispuesto en la legislación nacional, así como alentarles a que apliquen el Repertorio y las políticas y leyes nacionales que den efecto a sus disposiciones.

Legislación. Con el fin de erradicar la discriminación y lograr la prevención y protección social en el lugar de trabajo, en consulta con los interlocutores sociales y con expertos en $\mathrm{VIH} /$ sida, los poderes públicos deberían establecer el marco normativo adecuado y, cuando sea necesario, revisar las leyes laborales y otras medidas legislativas.

Asistencia. Los gobiernos deberían promover la asistencia mediante los programas de salud pública, el sistema de seguridad social y otras iniciativas. Deberían también adaptar y extender sus programas de prevención y asistencia al sector informal.

Niños y jóvenes. En los planes de erradicación del trabajo infantil, los gobiernos deberían tener muy en cuenta el impacto de la epidemia en los niños y los jóvenes que tienen enfermo al padre, a la madre o a ambos, o que han quedado huérfanos a causa del sida.

Trabajadores vulnerables. Los gobiernos deberían determinar cuáles son los grupos de trabajadores más vulnerables a la infección y adoptar estrategias que les permitan disminuir el riesgo de contraerla.

\section{Los empleadores, los trabajadores y sus organizaciones}

Normas en el lugar de trabajo. Los empleadores y los trabajadores deberían promover la concreción de normas y prácticas que impidan la propagación de la infección en el lugar de trabajo y protejan a los trabajadores infectados contra la discriminación.

Acuerdos. Los empleadores y los trabajadores deberían atenerse a la legislación y prácticas nacionales al negociar condiciones laborales y de contratación que tengan alguna relación con el VIH/ sida, y procurar incluir disposiciones de protección y prevención en los convenios nacionales, sectoriales y empresariales. 
Educación y capacitación. Los empleadores deberían establecer programas dirigidos a informar, educar y capacitar a los trabajadores en materia de prevención y atención del VIH/sida; inculcarles las normas pertinentes de la empresa, incluidas las destinadas a combatir la discriminación, y ponerlos al corriente de las prestaciones y derechos específicos del personal. Los trabajadores deberían aprovechar todos los medios disponibles para divulgar conocimientos sobre el VIH/sida y publicar material informativo actualizado sobre los derechos $\mathrm{y}$ prestaciones a su alcance.

Política de personal. Los empleadores y los trabajadores no deberían implantar ni consentir normas o prácticas discriminatorias contra los trabajadores infectados por el VIH o con sida y, más aún, deberían garantizar el derecho de las partes a denunciar ante la autoridad competente cualquier acto o conducta discriminatoria. Salvo casos excepcionalmente necesarios, los empleadores deberían abstenerse de solicitar pruebas o reconocimientos médicos relativos al VIH/sida. Asimismo, tendrían que garantizar y estimular la continuidad en el empleo de quienes estén infectados por el VIH o tengan una enfermedad derivada, mientras estén en condiciones de trabajar.

Confidencialidad. Toda información de un trabajador relativa al VIH/sida debería ser confidencial y constar únicamente en su expediente médico. El acceso a dicha información debería limitarse al personal médico, dándose a conocer solo cuando sea legalmente exigible o se cuente con el consentimiento de la persona afectada. En particular, los empleadores y las organizaciones de trabajadores no deberían tener acceso a datos personales referentes al VIH ni recabar por ningún medio esa información.

Reducción de riesgos. Los empleadores deberían garantizar un ambiente de trabajo sano y seguro, adoptando medidas de prevención tales como el suministro de equipo de protección personal y primeros auxilios, y brindando al personal sesiones informativas tendientes a modificar comportamientos de riesgo en relación con el VIH/sida. En los lugares donde los trabajadores entran en contacto regularmente con sangre humana y líquidos corporales, se han de tomar medidas suplementarias para que su personal conozca y aplique las precauciones estandarizadas y sepa cómo proceder si ocurre un incidente.

Acondicionamiento. Los empleadores deberían tomar medidas para adaptar, dentro de límites razonables, el lugar de trabajo a la situación de todo trabajador que padezca enfermedades derivadas del sida. Tales medidas podrían consistir en reordenar la jornada de trabajo, facilitar equipo especial, autorizar pausas de descanso, conceder tiempo libre para consultas médicas y licencias de enfermedad flexibles, prever el trabajo a tiempo parcial y establecer modalidades de reincorporación al trabajo.

Consultas y exámenes médicos. Los empleadores y los trabajadores deberían alentar y facilitar la realización de exámenes y consultas relativos al VIH/ sida de índole confidencial y voluntaria, que sean ofrecidos por personal sanitario especializado.

Impacto económico. Los empleadores, los trabajadores y sus organizaciones deberían realizar estudios que permitan determinar los efectos económicos del VIH/sida en el lugar de trabajo y desarrollar una estrategia adecuada a las condiciones locales que permita hacer frente a los mismos.

\section{PREVENCIÓN}

Los programas de información y educación en el lugar de trabajo son indispensables para combatir la propagación de la epidemia y fomentar una mayor tolerancia con los trabajadores que tienen el $\mathrm{VIH} /$ sida. Una labor didáctica eficaz puede ayudar a los trabajadores a protegerse contra la infección por VIH, paliar sensiblemente el oprobio y rechazo causados por esta enfermedad, reducir al mínimo las perturbaciones en el trabajo y lograr cambios de actitud y comportamiento muy favorables. Los programas deberían diseñarse y adaptarse en función de la edad, sexo, preferencias sexuales, características propias de cada sector y factores de riesgo. Se ha comprobado que la educación impartida por compañeros de trabajo y la intervención de personas infectadas por el VIH/sida resultan particularmente eficaces para la aplicación de estos programas.

\section{Campañas de información y educación}

Los programas de información deberían ir vinculados a campañas más generales sobre el $\mathrm{VIH} /$ sida en el plano regional y nacional. Tendrían que basarse en información precisa y actualizada sobre la forma en que se transmite - y no se transmite- el VIH, procurar erradicar los mitos existentes sobre el sida y explicar la manera de impedir la infección, los aspectos médicos de la enfermedad y las posibilidades en materia de cuidados y trata- 
miento. Convendría que estos cursos se den en horas de trabajo y que la participación de los trabajadores sea considerada una obligación profesional. Cuando sea apropiado los programas deberían:

- hacer hincapié en los comportamientos de alto riesgo y otros factores como la movilidad profesional, a consecuencia de los cuales algunas categorías de trabajadores se ven más expuestas que otras;

- facilitar información sobre la transmisión del VIH y la manera de reducir los riesgos;

- promover campañas centradas en los trabajadores jóvenes y en las mujeres;

- poner de relieve que el VIH no puede transmitirse por contactos ocasionales y que las personas infectadas no han de ser evitadas sino ayudadas, adaptando el trabajo a sus posibilidades;

- fomentar la higiene personal y una alimentación sana;

- promover prácticas sexuales más seguras.

\section{CAPACITACIÓN}

El mejor instructor es, a menudo, el propio personal, por lo que se recomienda que la educación, que debería formar parte del plan anual de capacitación en el lugar de trabajo, esté a cargo de compañeros y colegas en todos los niveles. Con este propósito, el personal encargado de la educación de colegas y compañeros debería recibir una capacitación que le permita:

- responder a las preguntas sobre la política con respecto al VIH/sida en el lugar de trabajo;

- conocer los métodos de prevención del VIH/sida;

- ayudar a sus compañeros a localizar los factores y hábitos que aumenten el riesgo de infección;

- aconsejar a los trabajadores afectados por el $\mathrm{VIH} /$ sida sobre cómo afrontar su situación y las consecuencias de la misma;

- identificar los comportamientos y prácticas que discriminan a los trabajadores con $\mathrm{VIH} /$ sida;

- asesorar sobre los servicios de salud y las prestaciones sociales existentes.

Es muy importante también que todos los trabajadores reciban adiestramiento sobre cómo proceder cuando ocurre un accidente en el lugar de trabajo, en particular:

- prestación de primeros auxilios;

- precauciones para reducir el riesgo de contacto con sangre humana y otros líquidos corporales, poniendo de relieve que la adopción de estas precauciones no depende de que haya alguna persona infectada por el VIH;
- uso del equipo de protección;

- derechos a indemnización en caso de accidente profesional.

\section{DETECCIÓN DEL VIH/SIDA}

Las pruebas de detección del VIH deberían efectuarse en establecimientos de salud y no en el lugar de trabajo, ya que en este último caso los resultados podrían ser divulgados y explotados indebidamente, vulnerándose la privacidad y la dignidad de los trabajadores. Incluso cuando se realizan fuera del lugar de trabajo, las pruebas relativas al VIH solo deberían ser efectuadas por personal especializado, con pleno consentimiento del interesado, respetándose estrictamente su carácter confidencial. Tampoco debería exigirse una prueba de detección del VIH en los regímenes nacionales de seguridad social, las pólizas de seguro general, los planes profesionales y el seguro de salud.

\section{Vigilancia epidemiológica}

Son admisibles las pruebas y sondeos epidemiológicos de detección del VIH efectuados de manera anónima e independiente en el lugar de trabajo, siempre que se realicen salvaguardando los derechos individuales y la confidencialidad. Puede ocurrir que los propios trabajadores deseen someterse a un examen médico, inclusive como parte de un programa de pruebas voluntarias. También estas pruebas deberían realizarse en los servicios locales de sanidad y no en el lugar de trabajo. No obstante, de existir servicios médicos adecuados en el lugar de trabajo y siempre que garanticen la debida confidencialidad, se podrá proceder a una prueba cuando el interesado dé su conformidad por escrito.

\section{RESPALDO Y ASISTENCIA}

La solidaridad y la asistencia médica son elementos fundamentales en todo lugar de trabajo. Es preciso promover una actitud de comprensión y apoyo a los trabajadores infectados, cerciorándose de que no son objeto de discriminación alguna. Con el fin de atenuar los efectos de la epidemia, habría que proporcionar asesoramiento $\mathrm{y}$ otras formas de apoyo social a los trabajadores afectados por el $\mathrm{VIH} /$ sida. La infección por el VIH no debería considerarse de un modo menos favorable que otras enfermedades o trastornos graves, especialmente en lo que se refiere a prestaciones, indemnizaciones y a una adaptación razonable del trabajo. Todos los trabajadores médicamente aptos para un empleo deberían gozar de estabilidad laboral y oportunidades de traslado y ascenso normales. 


\section{Servicios de salud y seguridad social}

Los gobiernos deberían velar por que las prestaciones estipuladas en la legislación se apliquen a los trabajadores con VIH/sida en las mismas condiciones que a los que padecen otras enfermedades graves. Debería estudiarse igualmente la viabilidad de establecer nuevas prestaciones para atender al carácter progresivo e intermitente del VIH/sida. Los empleadores, los trabajadores y sus organizaciones deberían colaborar con el gobierno en la adaptación de los sistemas de prestaciones existentes, con la inclusión de subvenciones a los salarios. También deberían tomar todas las medidas necesarias para que los trabajadores con $\mathrm{VIH} /$ sida y sus familiares no queden excluidos de la protección y de todas las prestaciones inherentes a los regímenes de seguridad social y a los planes profesionales.

\section{Programas de ayuda}

Podría resultar necesario establecer programas de ayuda o ampliar los existentes, con el fin de incluir diversos servicios que beneficien a los trabajadores infectados y sus familiares. Estos programas deberían tener presente que las mujeres son quienes normalmente se ocupan de atender a las personas enfermas de sida. Deberían satisfacer también las necesidades especiales de las mujeres embarazadas, así como las de los niños que se han quedado huérfanos a causa del sida y que pueden verse obligados a trabajar, quedando expuestos en mayor medida al abuso y la explotación sexuales. Los programas de ayuda a los pacientes y sus familias deberían contener:

- licencias por razones humanitarias;

- invitaciones a participar en programas de información y de educación;

- orientación hacia grupos de apoyo, entre ellos los de ayuda mutua;

- ayuda para que el trabajador o sus familiares puedan encontrar otro empleo;

- la adopción de medidas concretas (por ejemplo de apoyo escolar) para atender a las necesidades de los niños y jóvenes que han perdido a su padre, a su madre o a ambos;

- la concesión de ayuda monetaria directa o indirecta;

- asesoramiento médico y asistencia jurídica.

\section{PERTINENCIA DEL REPERTORIO}

La OIT ha dado un importante paso en la lucha contra el VIH/sida. Sus recomendaciones estableci- das en el Repertorio y dirigidas a gobiernos, empleadores y trabajadores de todo el mundo constituyen una herramienta útil para abordar de manera justa el problema del VIH/sida en el lugar de trabajo. Por ser el verdadero motor de la sociedad, el trabajo no puede permanecer ajeno a cuestiones de tanto impacto social. De ahí la insistencia de la OIT en coordinar las diversas políticas e iniciativas de lucha contra el sida en programas más amplios, nacionales e internacionales. En este sentido, es imprescindible conseguir un mayor compromiso de los diversos sectores involucrados con la salud y el trabajo, encaminado a paliar los efectos negativos de esta enfermedad. Mediante su Repertorio de recomendaciones prácticas sobre el $\mathrm{VIH} / \mathrm{sida}$, la OIT contribuye a salvaguardar los derechos y la dignidad de los trabajadores y de todas las personas infectadas por el VIH/sida.

\section{SYNOPSIS}

\section{HIV/AIDS in the workplace. Practical recommendations from the International Labor Organization}

There are now some 36 million people in the world infected with HIV/AIDS. It is estimated that more than 23 million of them are economically active, including 642000 persons in Latin America and the Caribbean. In the workplace, HIV/ AIDS reduces incomes, imposes added costs on companies, and undermines fundamental labor laws due to the discrimination and rejection that infected individuals suffer. In response, the International Labor Organization (ILO) has produced a document entitled An ILO code of practice on $\mathrm{HIV} / \mathrm{AIDS}$ and the world of work, which is summarized in this piece. The ILO document aims to help those in the workplace to cope with the HIV/AIDS epidemic through a set of guidelines related to: (a) preventing infection, (b) managing and reducing the impact that HIV/AIDS has on the workplace, (c) delivering care and support for infected workers and, in general, to all the people affected by this epidemic, and (d) eliminating discrimination against persons who are infected or are suspected of being infected. The ILO Code is intended to help in preparing and adopting specific measures in the workplace, thus promoting dialogue and other forms of cooperation among the government, employers, workers and their representatives, workplace health and safety officers, HIV/AIDS specialists, and other interested parties. The intention is also for the Code recommendations to be implemented and integrated with national laws, policies, and programs; company or business agreements; and workplace policies and action plans. This ILO Code is an important step in the struggle against HIV/AIDS. Aimed at governments, employers, and workers throughout the world, the Code recommendations constitute a useful tool in addressing the problem of HIV/AIDS in the workplace, in a just manner. As a "motor" of society, work cannot remain separated from issues of such great social impact. 